PERKIN AND WOOD: YELLOW COLOURING PRINCIPLES

\title{
XXXVIII.-Yellow Colouring Principles contained in various Tannin Matters. Part V. Pistacia len- tiscus, P. terebinthus, Tamaris africana, T. gallica, Ailanthus glandulosa, Ficus carica.
}

By Arthur George Perkin, F.R.S.E., and Percival John Wood.

Is previous communications, it was shown that numerous well-known tannin matters contain yellow colouring principles, which consist either of ellagic acid or members of the quercetin group. During the investigation of Sicilian sumach (Trans., 1896, 69, 1299), the attention of one of us was directed to the excessive adulteration that the commercial article is frequently subjected to, much care on this account being necessary to ensure that the material then examined was a pure sample of the leaves of the Rhus coriaria. It was interesting, how ever, to examine also authentic samples of the adulterants, could these be procured, and the present time was opportune, as we had promise of the aid of Prof. Procter on points of interest with reference to the tanning powers. Owing, apparently, to the desire of the Sicilians to keep the nature of these adulterants secret, attempts to procure them were at first a failure, although application to Sicily was made for us by large and well-known merchants in this country. Ultimately, we were fortunate in obtaining the aid of Mr. P. Gennadius, the Director of Agriculture of Cyprus, who readily supplied us with the required materials, for which our best thanks are due.

Owing to the excessive adulteration of sumach, the quantity exported from Palermo has continually decreased (Bulletin of Miscellaneous Information, Royal Gardens, Kew, Nov., 1895, p. 294), and this has been discussed in the Eco dei Campi. e. dei Boschi (Rome, Feb. 16, 1897, p. 99) and Bulletin de la Société nationale d'Acclimation (Paris, May, 1896). The adulteration consists in grinding with the sumach the leaves of other plants, principally those of Pistacia lentiscus, Ficus carica, Ailanthus glandulosa, Tamaris africana, and probably also Arctostaphylos $u v a$ ursi, and such a mixture, when ground, does not differ in appearance from ground sumach itself. With the aid of the microscope, however, it has lately been found possible to detect this adulteration to some extent, for, of the above plants, the leaves of the $R$. coriaria (sumach) alone are covered with minute, hair-like threads. The difficulty could be readily overcome by importing sumach in the unground leaf form only; any foreign admixture would then be at once visible. It is worthy of remark that Cyprus exports annually much unadulterated sumach in leaves. 


\section{Pistacia lentiscus (Mastic Tree).*}

The Pistacia lentiscus is a small tree about 20 feet high with evergreen leaves, which grows abundantly in most parts of Cyprus, where it is called "shinia." Mastic, or mastick, is the resin of the tree, and this is obtained by making transverse incisions in the bark. For some time, the leaves were exported to England by the Cyprus company, but now are hardly known in this country, although a considerable quantity is consumed at Lyons, in France, as an assistant dyeing material for silk stuffs. About 10,000 tons are exported from Tunis to Sicily annually at a price of $2 s$. per 100 kilos., and are re-exported from there (as sumach ?) at $3 s .7 d$. to $5 s .7 d$. for the same quantity. +

For the sample examined, we are indebted to the kindness of Mr. P. Gennadius, the Director of Agriculture of Cyprus. One thousand grams of the ground leaves were extracted with 10 kilos. of boiling water for 7 to 8 hours, and the light brown extract, which had the characteristic odour of mastic, was treated at the boiling temperature with a solution of 56 grams of lead acetate. The precipitate, which at first was yellow, became nearly colourless on digestion, and this precipitate, which is a lead compound of the tannin matter only, was removed by filtration and washed. On adding lead acetate to the filtrate, a permanent yellow precipitate was formed, and to this, ammonia was added, drop by drop, until the colour no longer increased in intensity; it was then collected, washed, and poured into dilute sulphuric acid, and, after the removal of lead sulphate, the reddish-brown liquid was repeatedly extracted with ether. On evaporating these extracts, a somewhat viscous residue was left, which, on treatment with boiling water, deposited yellow flocks; these were collected (the filtrate $A$ being reserved for examination) and purified by two or three crystallisations from dilute alcohol.

$0 \cdot 1147$ gave $0.2365 \mathrm{CO}$ and $0.0345 \mathrm{H}_{2} \mathrm{O} . \quad \mathrm{C}=56 \cdot 23 ; \mathrm{H}=3 \cdot 34$.

$$
\mathrm{C}_{15} \mathrm{H}_{10} \mathrm{O}_{8} \text { requires } \mathrm{C}=56.60 ; \mathrm{H}=3.14 \text { per cent. }
$$

In this manner, the yield of colouring matter was 1.5 grams, or 0.15 per cent. This was obtained as a lustrous mass of yellow needles, soluble in strong potassium hydroxide solution with a yellow coloration, which, on dilution, changed to a deep green. With aqueous lead acetate, it gave an orange-red precipitate, and reacted readily with mineral acids to form crystalline compounds.

It was acetylated in the usual manner, and the product, after being purified by crystallisation from alcohol, was obtained as a lustrous mass of colourless needles melting at $205-206^{\circ}$.

* Known in Sicily under the name of "Stinco."

† l'amphlet No. 1, Shinia Leaves, November, 1896, by P. G. Gennadius. 
$0 \cdot 1274$ gave $0 \cdot 2665 \mathrm{CO}_{2}$ and $0.0482 \mathrm{H}_{2} \mathrm{O} . \quad \mathrm{C}=57 \cdot 04 ; \mathrm{H}=4 \cdot 20$. $\mathrm{C}_{15} \mathrm{H}_{4} \mathrm{O}_{8}\left(\mathrm{C}_{2} \mathrm{H}_{3} \mathrm{O}\right)_{6}$ requires $\mathrm{C}=56.84 ; \mathrm{H}=3.86$ per cent.

From the colouring matter, by fusion with alkali, two products were obtained, melting respectively at $210^{\circ}$ and $238-240^{\circ}$. The former was found to be phloroglucinol, and the latter, which gave a blue-black coloration with ferric chloride, was evidently gallic acid.

The colouring matter of the leaves of Pistacia lentiscus is, therefore, identical with myricetin, which has been previously shown to exist in Rhus coriaria, or Sicilian sumach (Trans., 1896, 69, 1299), R. cotinus (Trans., 1897, 71, 1136), and the Myrica nagi (Trans., 1896, $69,1287)$.

The filtrate, A, from the crude colouring matter, was treated with sodium hydrogen carbonate and extracted with ether, which removed the last traces of myricetin, and the aqueous liquid was then neutralised with acid, again extracted with ether, and the extract evaporated. The brown, semi-crystalline residue was dissolved in water, and the solution saturated with salt, which caused the separation of a viscous product; this was removed by filtration, and from the filtrate, by means of ether, a crystalline acid was extracted which, after recrystallisation from water, were obtained in a colourless condition.

0.1100 gave $0.2002 \mathrm{CO}_{2}$ and $0.0360 \mathrm{H}_{2} \mathrm{O} . \quad \mathrm{C}=49 \cdot 63 ; \mathrm{H}=3.63$. $\mathrm{C}_{7} \mathrm{H}_{6} \mathrm{O}_{5}$ requires $\mathrm{C}=49.41 ; \mathrm{H}=3.53$ per cent.

This melted at $238-240^{\circ}$ with evolution of gas, gave with ferric chloride a blue-black coloration, and evidently consisted of gallic acid.

Judging from this result, and also from the fact that an aqueous extract of shinia leaves dyes calico mordanted with iron a blue-black shade, it appeared probable that the tannin which they contain was ordinary gallotannin. The deep, red-brown coloration produced on boiling an extract of the leaves with dilute sulphuric acid was, however, a property usually indicative of a catechol ratber than a gallotannin; moreover, some qualitative tests which Prof. Procter kindly carried out for us also hardly corroborated this view. Although, as mentioned in previous communications, it is not the intention in these papers to critically examine the tannin contained in the plants under examination, it was necessary, in this case, to make some search for the explanation of the above discrepancies.

The Tannin.-An alcoholic extract of the leaves was evaporated to a small bulk, and the deep-green liquid poured into water and repeatedly extracted with ether to remove chlorophyll, wax, and resinous matter. The aqueous solution was now extracted with ethylic acetate until no more tannin was dissolved, and the combined extracts were carefully evaporated; an orange-brown, friable product was thus obtained, readily soluble in water with formation of a pale brown solu- 
tion almost free from resinous and suspended matters. To the aqueous liquid, a little salt was added, the small quantity of precipitate thus formed removed, the filtrate extracted with ethylic acetate, and this solution evaporated to a small bulk. The addition of ether caused the precipitation of a trace of a brown product together with some tannin matter, and the now nearly colourless, supernatant liquid was carefully evaporated. A pale, intumescent, friable residue remained, having the appearance of a very pure gallotannic acid, but examination showed that it contained some yellow colouring matter, probably the glucoside of myricetin, which caused it to give, with iron salts, the reactions of a catechol tannin; it yielded, however, no precipitate with bromine water, which is characteristic of the latter. Not having sufficient material to effect its further purification by fractional precipitation with lead acetate, its behaviour with boiling dilute sulphuric acid was studied, for in this way catechol tannins usually form red anhydrides or phlobophanes, whereas gallotannin yields gallic acid. A small quantity of an orange-brown precipitate was formed, which, on investigation, proved to be crude myricetin ; this was removed by filtration, the filtrate extracted with ether, and the extract evaporated. The residue, after purification, formed colourless needles melting at $238-239^{\circ}$, which had the reactions of gallic acid. It thus appeared that the above product was gallotannic actd contaminated with the glucoside of myricetin.

The aqueous liquid which had been already extracted with ether still contained a considerable quantity of a tannin and also colouring matter, both probably as glucosides. To remove the latter, lead acetate was added to the solution as long as the precipitate formed was free from yellow colour after digestion at the boiling heat; for it has been shown in these communications that the main bulk of the tannin is thus thrown down before the glucosides of the yellow colouring matters, as these require for their precipitation an excess of the reagent. The precipitate was collected, washed, suspended in water, decomposed with sulphuretted hydrogen, and the lead sulphide removed by filtration. The filtrate, after repeated extraction with ethylic acetate, was evaporated to dryness, and an alcoholic solution of the brown residue treated with ether, until the supernatant liquid was almost colourless. This was decanted and slowly evaporated, there being thus obtained a pale brown, transparent, brittle product, which, when heated to $100^{\circ}$, apparently suffered some decomposition, in that it became of a much redder tint. With iron alum, an aqueous solution gave a blue-black coloration, turning greenish black on standing, but with bromine water no precipitate was formed. When digested with boiling dilute sulphuric acid, a reddish-brown precipitate separated, somewhat resembling a red anhydride or phlobophane; this was collected and 
washed three or four times with water, in which it was slightly soluble. To obtain some insight into the nature of the tannin, this product was fused with alkali, and this appeared to be a preferable mode of procedure, for should any glucoside of ordinary gallotannic acid originally have been present, which was likely, it must necessarily have been decomposed and the gallic acid thus formed removed by washing with water. The melt, dissolved in water and acidified, emitted a pungent odour of acetic acid, and phloroglucinol melting at $210^{\circ}$, and gallic acid melting at $238-240^{\circ}$, were isolated in the usual manner and recognised by the ordinary tests.

It seems apparent, therefore, that the leaves of Pistacia lentiscus contain gallotannic acid and a new tannin or tannin glucoside, yielding the above products on decomposition. As is well known, catechol tannins exist which, on fusion with alkali, form protocatechuic acid and phloroglucinol, of which quebrachotannic acid (Arata, Jahresbericht, 1879, $906)$ is an example, and the above result indicates as probable a natural group of these differing from one another by the number of hydroxyls which they contain. In dealing, however, with amorphous or apparently uncrystallisable substances of this class, a most exhaustive study is required to be sure of a correct interpretation of their properties, and therefore when a larger supply of the raw material is available, it is intended to wory thoroughly investigate the nature of this interesting product.

Tanning Properties.-According to Sir Thomas Wardle (private communication), the shinia leaves contain 11.29 per cent. of tannin. Under the direction of Professor Procter, a sample of the material employed in the above investigation was analysed by the International Conference method.

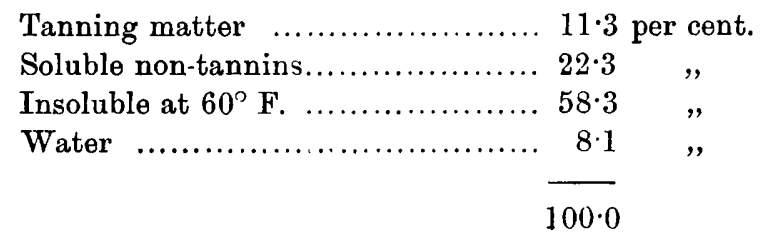

A good, plump leather is obtained from this material, but of a faintly reddish tint, the result being intermediate in character between those which are given by oak bark and sumach. Although evidently a useful tanning agent for dressing leather, it cannot be employed as a substitute for sumach, when a very light coloured leather is required, but in other respects it has very similar properties.

Experiments on the utility of shinia leaves as an assistant for the fixation of basic colouring matters upon cotton fabrics indicated, as was to 
be expected, that they have only half the strength of sumach. When twice the quantity was used, good results were obtained, although the paler shades had a greener and duller character; it seemed, therefore, that shinia leaves would be best employed for the production of the darker tints.

\section{Tamaris africana.}

This is a shrub or small tree characterised by its twiggy branches and minute scale-like leaves, which are largely used to adulterate sumach, and are known in Sicily under the name of "bruca." In the Kew Report (loc. cit.), it is described as having, in lieu of the leaf, prickly little shoots which thickly cover the lesser branches ; these are collected, ground, and mixed with the product of the true sumach plant. A very small sample of this material (140 grams) was obtained from Sicily, and although an endeavour was made to procure a larger supply from Tunis, where it is abundant, this met with no response.

For the isolation of the colouring matter, similar means were adopted to those employed with the leaves of the Pistacia lentiscus. The ethereal extract, on evaporation, gradually deposited minute, crystalline specks, and these could readily be distinguished in the dry, brown, viscous residue. The addition of hot water caused the separation of a yellow, flocculent precipitate which increased in quantity on cooling; this was collected, dried, and extracted with hot alcohol. A pale brown residue remained undissolved, consisting of the minute crystals above referred to; this was soluble in dilute alkali with a yellow colour, but was only sparingly soluble in the usual solvents. It was found to be ellagic acid, for not only did it dye identical shades, but gave, with nitric acid containing nitrous acid, the characteristic reaction of this substance.

The alcoholic extract, after concentration, was treated with water which caused the separation of yellow crystals; these were collected and purified by crystallisation from dilute alcohol. 0.4 gram was thus obtained.

$$
\begin{gathered}
0.0997 \text { gave } 0.2214 \mathrm{CO}_{2} \text { and } 0.0345 \mathrm{H}_{2} \mathrm{O} . \quad \mathrm{C}=60.56 ; \mathrm{H}=3.84 . \\
0.0966 \quad 0.2138 \mathrm{CO}_{2} \Rightarrow 0.0312 \mathrm{H}_{2} \mathrm{O} . \quad \mathrm{C}=60.36 ; \mathrm{H}=3.59 \\
\mathrm{C}_{16} \mathrm{H}_{12} \mathrm{O}_{7} \text { requires } \mathrm{C}=60.75 ; \mathrm{H}=3.79 \text { per cent. }
\end{gathered}
$$

It formed a glistening mass of yellow needles readily soluble in alcohol, and soluble in alkalis with a yellow coloration. With lead acetate, it gave an orange-red precipitate. Treated with mineral acids in the presence of acetic acid, it did not react, at least not so readily as is the case with quercetin and morin, and sufficient material was not available to compare its behaviour in this respect with rhamnetin. It dyed mordanted calico shades of a somewhat similar character to 
quercetin, but its properties could not be critically examined in this direction.

No further material from this plant was available for experiment; fortunately, however, the Tamaris gallica (see below) contained the same colouring matter, and this enabled us to gain further insight into its nature.

The aqueous filtrate from the above colouring matter was saturated with sodium hydrogen carbonate, extracted with ether to remove phenolic substances, neutralised with acid, and again extracted with ether. A crystalline acid was thus isolated, which, from its melting point and general properties, was found to consist of gallic acid.

An aqueous extract of the plant, when digested with dilute sulphuric acid at the boiling temperature, did not develop a red tint, as was the case with the shinia leaves, and which is usually a reaction of catechol tannins; it would thus appear that, in the Tamaris africana, only gallotannic and ellagitannic acids are present.

\section{Tamaris gallica.}

The Tamaris gallica, which in appearance closely resembles the Tamaris africana, flourishes in Cyprus, where the latter is not found. We are indebted to Mr. Gennadius for a sample of this plant. It weighed 170 grams, 50 of which were reserved for its tannin analysis.

Treated similarly to the Tramaris africana, it yielded ellagic and gallic acids, and contained, therefore, identical tannin matters. The yield of soluble yellow colouring matter was 0.6 gram.

$$
\begin{gathered}
0.0980 \text { gave } 0.2175 \mathrm{CO}_{2} \text { and } 0.0342 \mathrm{H}_{2} \mathrm{O} . \quad \mathrm{C}=60.53 ; \mathrm{H}=3.87 . \\
\mathrm{C}_{16} \mathrm{H}_{12} \mathrm{O}_{7} \text { requires } \mathrm{C}=60.75 ; \mathrm{H}=3.79 \text { per cent. }
\end{gathered}
$$

Its properties were identical with the yellow dye-stuff of the allied plant. Its unreactive nature with mineral acids suggested the presence of a methoxy-group, and this was found to be correct.

$0 \cdot 1483$ gave $0 \cdot 0810 \mathrm{AgI}$. $\mathrm{CH}_{3}=3 \cdot 48$.

$$
\mathrm{C}_{15} \mathrm{H}_{9} \mathrm{O}_{6}\left(\mathrm{OCH}_{3}\right) \text { requires } \mathrm{CH}_{3}=4.77 \text { per cent. }
$$

The acid residue from this experiment was treated with sodium hydrogen sulphite solution, and the suspended yellow precipitate collected and crystallised from dilute alcohol. The acetyl compound, prepared from a trace of this substance, formed colourless needles melting at $189-191^{\circ}$.

0.0831 gave $0 \cdot 1820 \mathrm{CO}_{2}$ and $0 \cdot 0277 \mathrm{H}_{2} \mathrm{O} . \quad \mathrm{C}=59 \cdot 72 ; \mathrm{H}=3 \cdot 70$. $\mathrm{C}_{15} \mathrm{H}_{10} \mathrm{O}_{7}$ requires $\mathrm{C}=59.60 ; \mathrm{H}=3.31$ per cent.

Fused with alkali, the methyl ether yielded an acid melting at $194-196^{\circ}$ which, with ferric chloride, gave a green coloration, and was 
evidently protscatechuic acid. Distinct evidence was also obtained of the presence of phloroglucinol.

These results indicate that the colouring matter of the Tamaris africana and $T$. gallica contains a quercetin monomethyl ether. This does not appear to be either isorhamnetin or rhamnetin, for these methyl ethers of quercetin are distinguished by their sparing solubility in alcohol, and it is therefore most probably a new substance. Whether the product obtained above is homogeneous or contains also quercetin could not be determived; the methoxy-determination favours this supposition, but, on the other hand, low results are sometimes given with this class of substance by Zeisel's method.

On acetylising a trace of the methyl ether in the usual manner, colourless needles were obtained melting at $169-171^{\circ}$.

Attempts will be made in the ensuing autumn to procure a large supply of the plant for an exhaustive examination of this colouring matter.

A tannin analysis carried out under the direction of Professor Procter gave the following result.

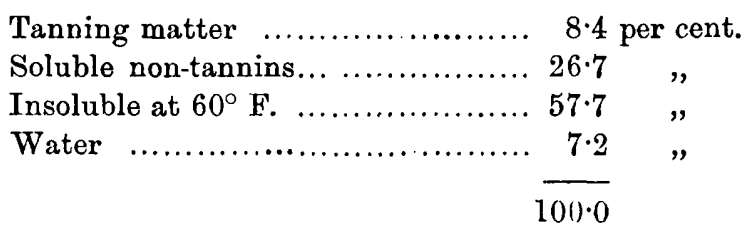

This sample was collected in November, which is not a good season from a tanning point of view, and it is probable, therefore, that the tannin content of that procured from June to August, will be somewhat greater.

\section{Ailanthus glandulosa.}

This is a tree of large size and handsome appearance, bearing numerous pinnate leaves from one to two feet long or more. It is a native of India and China, but is common on the Continent, where it is frequently reared for the shading of public walks. For the sample of leaves we are indebted to Mr. Gennadius, of Cyprus.

The finely ground material was extracted with boiling water, and the extract treated for the isolation of the colouring matter by methods similar to those already described. On evaporating the ethereal extract, a viscous residue was left, interspersed with fine crystals, and from this, on treatment with boiling water, brownish-yellow flocks of a colouring matter separated. These were collected and extracted with boiling alcohol, which left undissolved a brownish, crystalline powder having the dyeing and other properties of ellagic acid. 
The alcoholic extract, on treatment with boiling water, deposited crystals of a colouring matter, on cooling, but in a very impure condition. The product was therefore poured into water, treated with excess of sodium hydrogen carbonate, the mixture extracted with ether, and the extract evaporated. It was finally purified by crystallisation from dilute alcohol.

$$
\begin{gathered}
0.1068 \text { gave } 0.2335 \mathrm{CO}_{2} \text { and } 0.0385 \mathrm{H}_{2} \mathrm{O} . \quad \mathrm{C}=59.62 ; \mathrm{H}=4.00 . \\
\mathrm{C}_{15} \mathrm{H}_{10} \mathrm{O}_{7} \text { requires } \mathrm{C}=59 \cdot 60 ; \mathrm{H}=3.31 \text { per cent. }
\end{gathered}
$$

It formed a mass of yellow needles, soluble in alkalis with a yellow colour and giving an orange-red precipitate with lead acetate. The acetyl compound crystallised in colourless needles melting at $189-191^{\circ}$.

0.1149 gave $0.2453 \mathrm{CO}_{2}$ and $0.0 .455 \mathrm{H}_{2} \mathrm{O} . \quad \mathrm{C}=58.22 ; \mathrm{H}=4.39$. $\mathrm{C}_{15} \mathrm{H}_{5} \mathrm{O}_{7}\left(\mathrm{C}_{2} \mathrm{H}_{3} \mathrm{O}\right)_{5}$ requires $\mathrm{C}=58.59 ; \mathrm{H}=3.90$ per cent.

On fusion with alkali, protocatechuic acid (m. p. 194-196 ${ }^{\circ}$ ) and phloroglucinol (m. p. $210^{\circ}$ ) were identified as the principal products. The colouring matter of Ailanthus glandulosa is therefore quercetin, and to this and ellagic acid, its dyeing properties must be assigned.

The aqueous filtrate from the quercetin was found to contain a large quantity of gallic acid, which was identified by its melting point, $238-240^{\circ}$, and general properties. The tannin of the Ailanthus glandulosa is evidently gallotannic acid, for the dyeing properties of the leaves with mordants, and the behaviour of the aqueous extract with acids, corroborate this view.

A tannin analysis carried out under the direction of Professor Procter gave the following result.

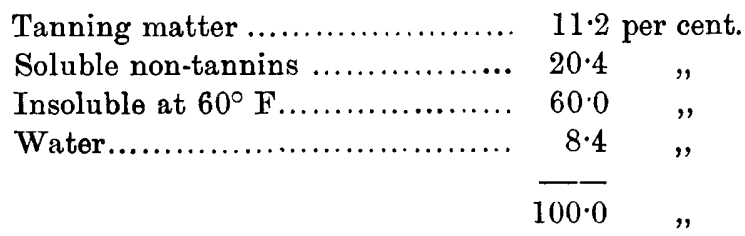

Curiously enough, although so high a percentage of tanning matter is apparently present, leather is scarcely tanned by an extract of these leaves, but is stained a dull dirty colour. They are, therefore, of little utility for tanning purposes, and as an adulterant of sumach would exert a deleterious influence.

\section{Ficus carica.}

For a supply of the leaves of this plant, the common fig tree, we are indebted to the kindness of Mr. P. Gennadius. An aqueous 
extract dyed alum mordanted calico but faintly yellow, indicating only a trace of colouring matter, and this was corroborated by experiments carried out according to the methods previously employed. From 2000 grams, only 0.08 gram of a very impure colouring matter was obtained, having dyeing properties analogous to those of quercetin ; unfortunately, its identity could not be established, for an attempt to prepare its acetyl derivative failed on account of the impurity present.

The filtrate from the colouring matter contained no gallic acid, and the dyeing property of the leaves with iron mordanted calico indicated an almost entire absence of tannin.

A tanning analysis carried out under the direction of Prof. Procter gave the following result.

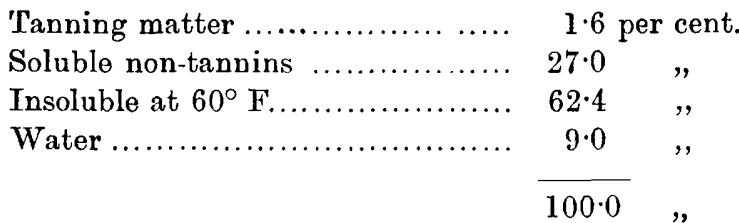

Skin remained untanned in an extract of these leaves, and was stained a dirty olive colour. It would appear to be an extremely deleterious adulterant of sumach.

\section{Gambuzzo.}

In Sicily, this name is given to the small stalks branching from the main root of the Rhus coriaria, which are ground to powder and admixed with sumach. We are indebted to Messrs. Kuypers, Ostler, and Scott, of Hull, for a sample of this material. It has been previously shown by one of us (Trans., 1897, 71, 1136) that the leaves of the $R$. cotinus contain myricetin, whereas it is well known that the stem (young fustic) contains fisetin, and it was thus interesting to determine if this also was the case with the $R$. coriaria.

The stems of the latter possessed but feeble dyeing power, and only after the extraction of much material was a trace of the yellow colouring matter isolated. This on examination was found to be myricetin, and not fisetin, for it was readily identified by its characteristic colour reactions with dilute potassium hydroxide solution.

The aqueous filtrate from the colouring matter contained a considerable quantity of gallic acid, indicating the presence of gallotannic acid in this portion of the plant also.

The above results show that, whereas the leaves of Pistacia lentiscus and the "gambuzzo" contain myricetin, the colouring matter of sumach ( $R$. coriaria), the leaves of Ailanthus glandulosa and Tamaris africana contain respectively quercetin and one of its methyl ethers. 


\section{4}

PERKIN AND WOOD : YELLOW COLOURING PRINCIPIAS

It seemed possible, therefore, that an admixture of the latter with sumach might be detected by the examination of the yellow colouring matter present in the material, for the acetyl compound of such a mixture should have a depressed or indefinite melting point. Experiments carried out in this direction with mixtures of pure quercetin and myricetin, however, hardly favoured the utility of such a method, unless very excessive adulteration had been employed. Thus $(a)$ equal parts of myricetin and quercetin yielded an acetyl compound which, after two crystallisations from alcohol, melted at $195-198^{\circ}$, whereas (b) a similar derivative from three parts of quercetin and one part of myricetin melted at $178-182^{\circ}$. As acetyl myricetin melts at $204-206^{\circ}$, the depression of the melting point to $195-198^{\circ}$ in $(a)$ is small, and it became evident that, owing to the more sparing solubility of acetylmyricetin in alcohol, the latter, if present in quantity, could be obtained free from acetylquercetin by frequent recrystallisation.

\section{Broach Leaves.}

These constitute a valuable tanning agent which is employed in South Africa as a substitute for sumach, and in lieu of the leaves of the Colpoon compressum (Cape sumach) (Trans., 1897, 71, 1132) which are becoming scarce owing probably to injudicious gathering and lack of care of the plant. Information as to the botanical origin of these leaves is at present lacking, but they are said to be derived from a small shrub flourishing in the same localities and side by side with the Colpoon compressum. Mr. R. H. Coaton, to whom we are indebted for our supply of material, 500 grams, promises fuller information on his return to the Cape.

The finely ground leaves were extracted with boiling water, the extract treated with lead acetate solution, and the light yellowish precipitate collected and treated for the isolation of the colouring matter according to the methods previously employed in this paper. The residue from the ethereal extract, when diluted with water, yielded a yellow, flocculent precipitate of the colouring matter, which was purified by crystallisation from alcohol; 0.5 gram was thus obtained.

$$
\begin{gathered}
0.0911 \text { gave } 0.1992 \mathrm{CO}_{2} \text { and } 0.0310 \mathrm{H}_{2} \mathrm{O} . \quad \mathrm{C}=59.62 ; \mathrm{H}=3.78 . \\
\mathrm{C}_{15} \mathrm{H}_{10} \mathrm{O}_{7} \text { requires } \mathrm{C}=59.60 ; \mathrm{H}=3.31 \text { per cent. }
\end{gathered}
$$

It formed glistening, yellow needles closely resembling quercetin in appearance, but soluble in dilute potash with a deep green coloration, evidently the result of oxidation; with the concentrated alkali, a yellow solution was obtained, becoming green only on dilution. On acetylisation, an acetyl compound was obtained, crystallising in colourless needles and melting at $188-190^{\circ}$, and by fusion with alkali 
protocatechuic acid and phloroglucinol were identified as the main decomposition products.

This colouring matter is evidently identical with that contained in the Arctostaphylos uva ursi, a short account of which has been already communicated to the Society (Proc., 1898, p. 104), and which so closely resembles quercetin except in its behaviour towards dilute alkali. In this case also, attempts to remove an impurity having this peculiar property were unsuccessful, and this is remarkable, for if due to such a cause the amount present must be of considerable quantity.

The aqueous filtrate from the colouring matter contained no gallic acid, and the behaviour of the aqueous extract of the leaves towards boiling dilute sulphuric acid, whereby a deep red liquid was produced, indicated the presence of a catechol tannin. This was confirmed by further tests with bromine water and iron alum solution in the usual manner, for these respectively yielded a precipitate and green-black coloration.

Mr. R. H. Coaton has kindly furnished us with the following analysis of this tannin matter.

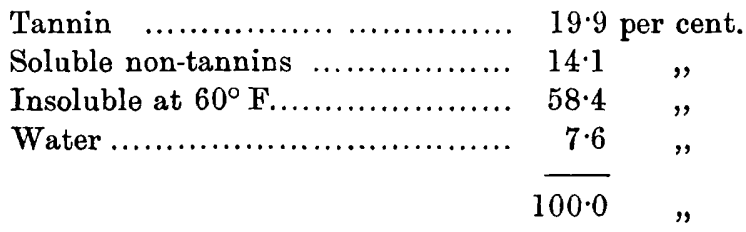

The galls of Pistacia terebinthus were examined for yellow colouring matter. These, which are of a peculiar horn-like shape, are caused by the punctures of insects, and constitute a very valuable tanning material. Owing, however, to an uncertainty in the supply, they appear to have fallen into disfavour in this country.

From 100 grams of these, but a trace of yellow colouring matter was with difficulty isolated, which, judging from its colour reactions with dilute potash solution, appeared to be myricetin. Dyeing experiments with mordanted calico also corroborated this view.

An investigation has also been carried out on the colouring matter of "arbutine," a ground leaf substitute for sumach. It was found to contain quercetin, and, judging from the gallic acid formed during the isolation of the dye-stuff, also gallotannic acid. Unfortunately, the botanical origin of these leaves was not forthcoming on inquiry, and thus no details of the work are at present given. Possibly they are derived from the Arbutus unedo, which has been employed in Greece and southern Europe as a tanning material.

In mangrove cutch, a well-known tanning material obtained from the Ceriops candolleana, yellow colouring matter was found to be 
absent. Judging from its resemblance to catechutannic acid, it was examined for catechin, but experiments in this direction were also unsuccessful.

We are much indebted to Prof. Procter for his aid during this investigation, and also for his assistance in obtaining the necessary material.

\author{
Clothworkers' Research Laboratory, \\ Dyeing Department, \\ Yorkshire College.
}

